\title{
A nine-year clinico-epidemiological study of Histoid Hansen in India
}

\author{
Vibhu Mendiratta, Arpita Jain, Ram Chander, Anamita Khan, Meenu Barara \\ Department of Dermatology, Venereology \& Leprosy, Lady Hardinge Medical College, New Delhi, India
}

\begin{abstract}
Introduction: Histoid Hansen is rare but a well-defined entity with specific clinical, histopathological, and bacteriological features. The incidence has been reported to vary from 1-2\% amongst total leprosy patients.

Methodology: We performed a retrospective analysis of the records of patients identified as histoid Hansen on the basis of clinicohistopathological criteria, who attended the Hansen clinic from 2000-2009.

Results: A total of 962 leprosy patients attended the Hansen clinic from 2000-2009, and of these, 11 (1.14\%) were diagnosed as histoid Hansen. The youngest patient on record was 14 years of age. Nearly $70 \%$ of the patients were untreated and the rest were on multidrug therapy (MDT) before development of histoid lesions. Three patients showed Erythema nodusum leposum (ENL) reaction.

Conclusion: India has achieved elimination of leprosy, but new cases of histoid are still recorded with the same incidence rate. Reactions are not uncommon in such forms, and may be a transition phase to manifest lepromatous leprosy. A thorough knowledge and early detection is needed for this uncommon variant of leprosy which can pose a threat to our elimination drive.
\end{abstract}

Key words: histoid Hansen; leprosy; India; epidemiology

J Infect Dev Ctries 2011; 5(2):128-131.

(Received 12 May 2010 - Accepted 20 July 2010)

Copyright (C) 2011 Mendiratta et al. This is an open-access article distributed under the Creative Commons Attribution License, which permits unrestricted use, distribution, and reproduction in any medium, provided the original work is properly cited.

\section{Introduction}

Histoid Hansen is a rare, but well-defined entity with specific clinical, histopathological, and bacteriological features. It was originally described by Wade in 1963 as discrete, firm lesions and domeshaped nodules which develop on apparently normal skin in patients with lepromatous leprosy [1]. Its exact etiopathogenesis is not well understood as it may arise de novo or may develop after an inadequate and irregular treatment with dapsone monotherapy or multidrug therapy (MDT) [2]. The incidence has been reported to vary from 1-2\% among total leprosy patients [3].

Histoid leprosy might represent an enhanced response of the multibacillary disease in localizing the disease process. An increase in both cell mediated and humoral immunity against mycobacterium leprae, as against in lepromatous leprosy, has been hypothesized, [4]. Since then there have been many case reports of histoid Hansen with variable presentation [5-7].

Considering the rarity of histoid leprosy, as well as its variable clinical expression and uncertainty regarding its etiopathogenesis, we reviewed the data of histoid Hansen patients who attended our Hansen clinic from July 2000 to June 2009.

\section{Methodology}

A retrospective as well as prospective analysis of the leprosy records of the patients diagnosed as histoid Hansen on the basis of clinichistopathological criteria, who attended the Hansen clinic at Lady Hardinge Medical College, New Delhi, from July 2000 to June 2009 was performed. Their records were analyzed for their demographic profile, duration of leprosy, duration of histoid lesions, type and distribution of histoid lesions, number of nerves enlarged, presence of any reaction and the treatment taken by the patients. Bacteriological index, morphological index and details of the histopathological findings were noted. The data was pooled and analyzed for the above parameters.

\section{Results}

A total of 962 leprosy patients attended the Hansen clinic from July 2000 to June 2009. Eleven cases were diagnosed as histoid leprosy, resulting in a prevalence of $1.14 \%$. There were nine $(81 \%)$ males and two $(18 \%)$ females, making a male to female 
Figure 1. Ulceration of the histoid papule.

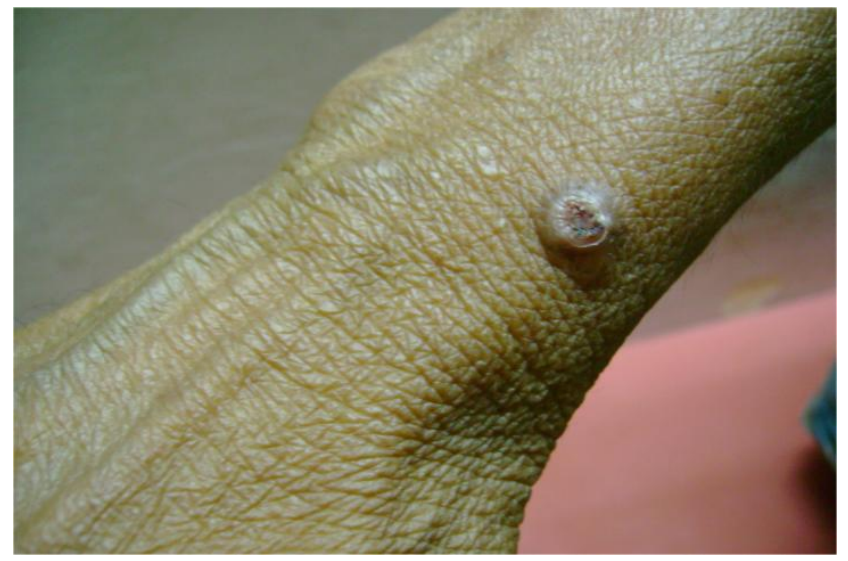

ratio of $4.5: 1$. Nine patients $(80 \%)$ were between 20 and 40 years of age. The mean age affected was 30.7 years. The youngest of was 14 years old, and one patient was 55 years of age. Only three patients $(30 \%)$ had history of contact with a person with leprosy. Most of the patients $(70 \%)$ were manual laborers and nearly $80 \%$ had history of residence in the endemic states of Bihar and Uttar Pradesh.

Six patients $(54 \%)$ were de novo cases of histoid leprosy, in four of whom leprosy first manifested in the lepromatous leprosy (LL) form and in the other two patients histoid morphology was the only observed pattern present. Four patients (36\%) were defaulter cases of LL Hansen. One of them had taken dapsone monotherapy for two years and manifested histoid after nearly 20 years of therapy. The other three were defaulters on MDT regimen. One patient had borderline leprosy (BL) Hansen and was still on MDT when her histoid morphology manifested. Those who had completed their two years of MDT therapy before the appearance of histoid lesions were disease-free for periods varying from three years to 14 years.

All the patients presented with smooth, shiny, succulent nodules overlying a normal layer of skin. In three patients $(30 \%)$, the nodules were uncountable in number, while five patients $(40 \%)$ had 20 to 40 nodules and the remaining three patients $(30 \%)$ had less than 20 lesions. Eight patients (70\%) also had papules amidst the nodules. Ulceration of the nodules was seen in one patient (Figure1). Remarkably, all $(100 \%)$ patients had facial involvement in the form of papules and nodules mostly over the central part of the face involving the forehead, nose, lips, cheek and chin. Backs and buttocks were the next most common sites involved (80\%), and half of the patients presented with papules and nodules over their extremities (Figure 2). None of the patients had mucosal involvement.

Two or more peripheral nerves were involved in all the cases, most common being the ulnar followed by the lateral popliteal nerves. Additionally, three (25\%) of the patients had grade I deformity.

A slit smear and histopathological examination was performed in all the cases. Histopathology was suggestive of histoid in all of them. The smear showed abundant long, slender, acid-fast staining bacilli with tapering ends in most of the fields. The bacteriological index of the slit smear varied from $5+$ to $6+$ (mean 5.7) with the presence of globi in a few. The morphological index varied from $10 \%$ to $45 \%$ (mean 35.4). In three prospective patients, a slit smear was also taken from a distant patch of apparently normal-looking skin. The sample was negative in one patient but the other two samples were $1+$ and $3+$ respectively in the other two patients.

Three out of 11 patients experienced type II reaction during the course of their disease.

All the patients were put on MDT and responded well to treatment. Nine of our patients successfully completed two years of MDT and two patients are still on therapy. Successful follow-up was possible in all of the eight cases released from treatment for only two to four years. All of them showed progressive decline in their bacillary index $(\mathrm{BI})$, decreasing from $5+$ or $6+$ to $4+$ or zero. One patient returned with relapse as a $\mathrm{BL}$ case five years after stopping of MDT. His BI had reduced from $6+$ to only $4+$ in five years.

Figure 2. Histoid nodules over the extensor aspect of elbow.

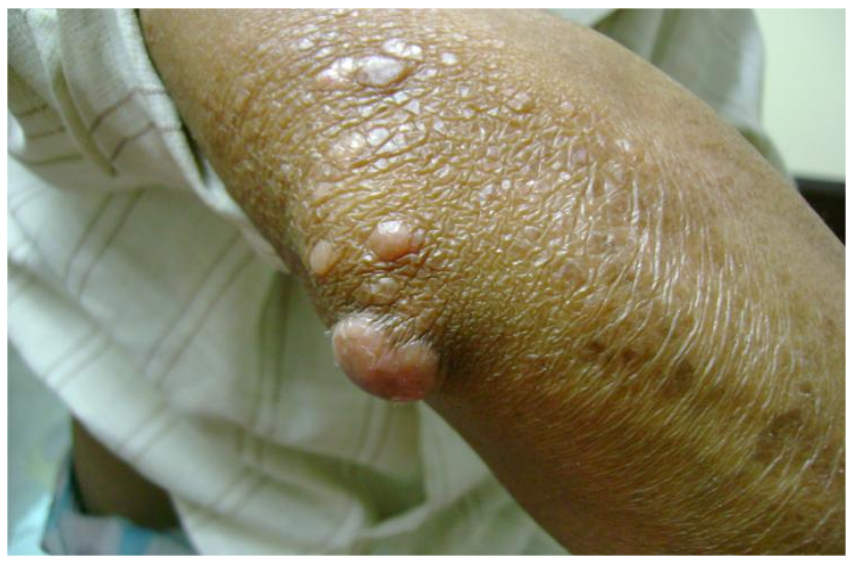

\section{Discussion}

Ever since it was first reported by Wade in 1963 [1], histoid leprosy has remained enigmatic. It is a very rare form of multibacillary leprosy with 
Figure 3. Yearly trend of histoid Hansen cases in 9 years in our outpatient department

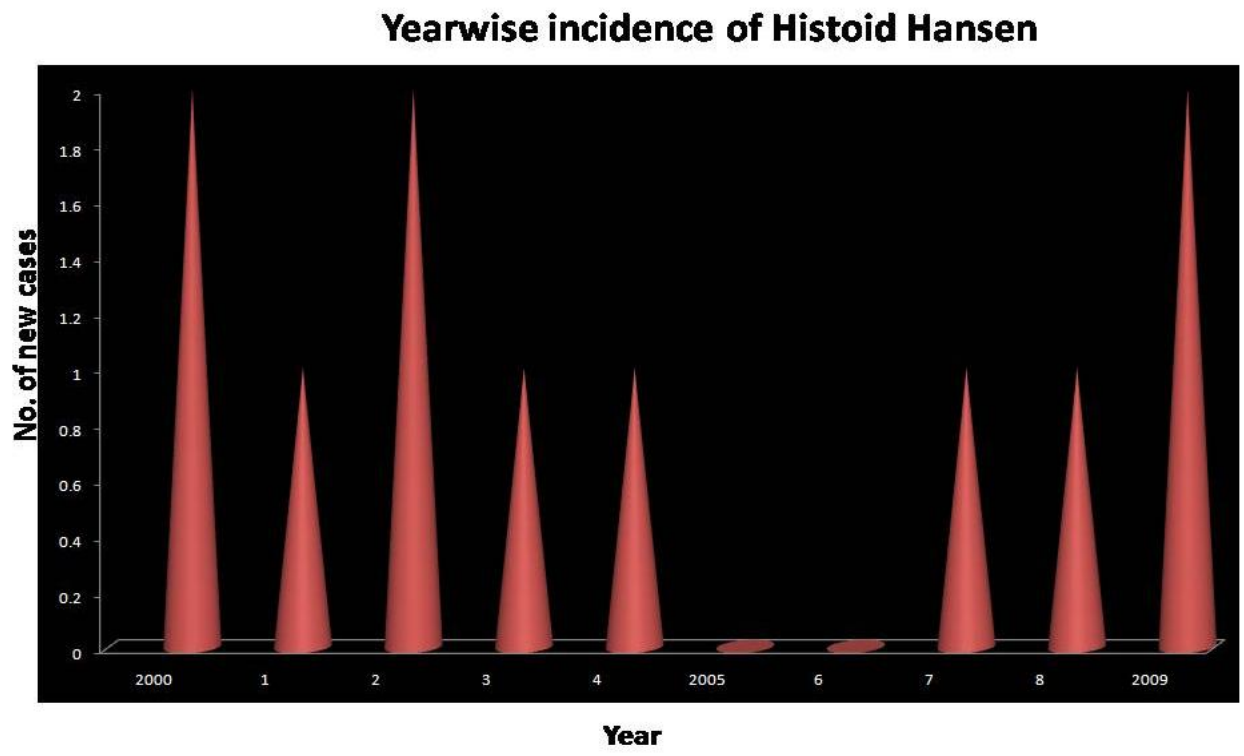

characteristic clinical, bacteriological, and histopathological features. This form of leprosy derives its name from its histopathological appearance, which is similar to that of a tumor derived from single element, namely spindle-shaped histiocytes [8]. Though previously thought to arise after long-term monotherapy with dapsone or an irregular and incomplete therapy, it is now known to occur as a relapse after complete MDT or even as a de novo form, without any previous history of leprosy therapy $[2,9,10]$.

There are very few previous studies on this form of leprosy. Therefore, we conducted a retrospective analysis of all the histoid leprosy patients registered with us between 2000 and 2009. During the course of the study, an additional 3 new cases were prospectively diagnosed. The prevalence of histoid in our study was $1.14 \%$, which was similar to that recorded in previous studies [3].

Most of the patients were between 16 and 35 years of age. This observation was similar to the findings in other studies [7,10,11]. One adolescent (14 years old) also showed features of the histoid form in our series, whereas Kalla et al. [11] have still recorded the youngest patient (8 years old). The male to female ratio (4.5:1) in our study was nearly in accordance to the 5.7:1 ratio recorded by Kaur et al. [10], but lower than that noted by Sehgal and Srivastava (8.2:1) [7]. The increased incidence in males points towards their greater exposure, due to outdoor work. This speculation can also be corroborated by the fact that $70 \%$ of our patients $(8$ out of 11) were manual laborers.

The typical clinical features of histoid were present in all the patients, including numerous shiny, succulent, smooth, dome-shaped, non tender, soft to firm nodules overlying normal looking skin, along with papules and plaques. It commonly affects the buttocks, back, face and extremities. Noticeably, the face was involved in all 11 of our patients, affecting mostly the central-facial area involving forehead, tip of nose, cheeks and chin. This observation does not concur with the observations of other studies, in which the buttocks are the commonest site of presentation [10]. Genital and mucosal lesions have been described by some authors, but none of our patients had any such involvement [12].

It was earlier believed that reactions are very uncommon in histoid cases. Contrarily, three out of 11 of our patients $(27 \%)$ had an episode of ENL either prior to or during therapy. Forty per cent (16 out of 40) had been recorded by Kaur et al. [10], and a few cases have been recorded by others [4,11]. All the patients who developed ENL reactions were a prior case of LL Hansen and the reactional episodes were followed by the development of histoid morphology in all of them. This trend strengthens the hypothesis drawn by Kaur et al. [10] that ENL can be a commonly observed phenomenon in a given patient during transition to manifest histoid. Only one of our patients showed recurrent ENL reaction after labeling him as a histoid case. 
Histoid was earlier considered to occur only in patients who took long-term monotherapy with dapsone [1,2]. Later, reports after MDT therapy and de novo occurrence were also recorded [10]. Histoid was known to occur commonly in patients who had previously manifested with LL lesions. We had two patients who developed histoid leprosy without any previous history or features of LL or any form of leprosy. Similar findings have also been recorded by Sehgal et al. and Kaur et al. [9,10] and Sehgal has rightly suggested that histoid could be a distinct form of leprosy and should not always be considered a variant of LL Hansen.

Histoid leprosy is treated as a multibacillary disease. We could not follow up our patients for more than four years. One of our patients showed a very slow decline in his BI after two years of MDT (from $6+$ to $4+$ in 4 years) and later relapsed as BL leprosy after five years of treatment completion. Whether this outcome was due to the resistant form of his bacilli could not be confirmed by sensitivity tests, but it raises the serious consideration that two years of MDT may not be sufficient for such highly bacillated forms. Today only one year of therapy is recommended for multibacillary forms and attempts are being made to further reduce the duration. However, whether histoid should be treated as other multibacillary forms or whether other immunotherapies should be added to the treatment regimen deserves consideration. Kaur et al. [10] proposed that relapses could be due to re-infection or due to multiplication of dormant resistant bacilli. The second possibility seemed to apply to our patient as re-infection can be ruled out, keeping in mind the continued persistence of his BI to 4+ even after five years of follow-up. We could not find any prior reports of relapse in a treated histoid case.

In January 2007, India was declared to have eliminated leprosy. We have seen a constant trend of histoid Hansen in our department, varying from 0-2 cases every year (Figure 3). After 2007, three cases of histoid were diagnosed in our department; furthermore, cases are also still being reported from areas, including those with low leprosy endemicity [13]. As suggested by Palit and Inamadar, histoid form could serve as a reservoir of leprosy and as a source of new cases. This could pose a serious threat to our elimination program.
Though presentation of leprosy has been shown to decrease at the primary health care level, due to its near elimination; multibacillated forms will still continue to present in our speciality especially at a tertiary care level. histoid Hansen can be easily identified and should be promptly treated to prevent the further spread of the disease in society.

\section{References}

1. Wade HW (1963) The histoid variety of lepromatous leprosy. Int J Lepr 31: 129-43.

2. Rodriguez JN (1969) The histoid leproma: its clinical characteristics and significance. Int J Lepr 37: 1-21.

3. Sehgal VN and Srivastava G (2004) Histoid Leprosy. 3rd ed. New Delhi: Jaypee Brothers Medical Publishers, 12.

4. Sehgal VN and Srivastava G (1987) Status of histoid leprosy - a clinical, bacteriological, histopathological and immunological appraisal. J Dermatol (Tokyo) 14: 38-42.

5. Bhutani LK, Bedi TR, Malhotra YK, Kandhari KC, Deo MG (1974) Histoid leprosy in north India. Int J Lepr 42: 174-181.

6. Salodkar AD and Kalla G (1995) A clinic-epidemiological study of leprosy in northwest Rajastjan: Jodhpur. Ind J Lepr 62: 161-166.

7. Sehgal VN and Srivastava G (1988) Histoid leprosy: a prospective diagnostic study in 38 patients. Dermatologica III: 212-217.

8. Ewing J (1942) Neoplastic diseases. A treatise on tumors. 4th edition. Philadelphia and London: W.B. Saunders Company. 33 p.

9. Sehgal VN, Aggarwal A, Srivastava G, Sharma N, Sharma S (2005) Evolution of histoid leprosy (de novo) in lepromatous (multibacillary) leprosy. Int J Dermatol 44: 576-578.

10. Kaur L, Dogra S, De D Saikia UN (2009) Histoid leprosy: a retrospective study of 40 cases from India. Br J Dermatol 160: 305-310.

11. Kalla G, Purohit S, Vyas MC (2000) Histoid, a clinical variant of multibacillary leprosy: report from so called endemic areas. Int J Lepr Other mycobact Dis 68: 267-271.

12. Nigam PK and Singh G (1990) Mucosal and genital lesions in histoid leprosy. Int J Dermatol 29: 207-208.

13. Palit A and Inamadar AC (2007) Histoid leprosy as reservoir of disease: a challenge to leprosy elimination. Lepr Rev 78: 47-49.

\section{Corresponding author}

Dr. Arpita Jain

Department of Dermatology, Leprosy and STD

Lady Hardinge Medical College

New Delhi

India

Telephone: +919891883881

Email: arpitajain1@gmail.com

Conflict of interests: No conflict of interests is declared. 